\title{
A Compact Temperature Sensor Based on Micrometric Optical Fiber Coupler Tip
}

\author{
Ming Ding, ${ }^{1 *}$ Pengfei Wang, ${ }^{1,2}$ Gilberto Brambilla ${ }^{1}$ \\ ${ }^{1}$ Optoelectronics Research Centre, University of Southampton, Southampton, SO17 1BJ, UK \\ ${ }^{2}$ Photonics Research Centre, Dublin Institute of Technology, Kevin Street, Dublin 8, Ireland \\ * md20g09@orc.soton.ac.uk
}

\begin{abstract}
A compact temperature sensor based on a coupler tip with micrometric size is demonstrated. This sensor can measure a temperatures as high as $1283{ }^{\circ} \mathrm{C}$ with an average sensitivity of $\sim 12 \mathrm{pm} /{ }^{\circ} \mathrm{C}$.

OCIS codes: (230.1150) All-optical devices; (060.2340) Fiber optics components; (060.1810) Buffers, couplers, routers, switches, and multiplexers; (060.2370) Fiber optics sensors.
\end{abstract}

\section{Introduction}

In the last decade, the use of optical fibers for temperature monitoring has been widely investigated [1-4] because of their immunity to electromagnetic interference and possibility to work in contact with explosives. Among optical fiber devices, fiber Bragg gratings (FBGs) $[1,5,6]$ are possibly the most common tool used for temperature sensing: although they have been used for temperature measurements higher than $1200^{\circ} \mathrm{C}$ and benefit from wavelength encoding, their fabrication requires expensive laser set-ups and manufacturing processes, like hydrogen loading and annealing.

This paper presents a temperature sensor based on a coupler tip (Fig. 1). Optical fiber couplers can be used as a highly sensitive thermometers as in the coupling region modal beating depends both on the coupling region length and its refractive index, thus on its temperature. In conventional optical fiber couplers, with cross sections in excess of $10 \mu \mathrm{m}$ and coupling lengths limited to few $\mathrm{mm}$, the transmission spectrum presents dips with a periodicity typically of the order of few hundred nanometers: a small temperature increase results in a proportionally small spectral shift, thus small responsivity [7]. On the contrary, in micrometric fiber coupler tips (MFCTs), this periodicity is of the order of few/tens of nm: a wavelength shift of few nanometers generally results in large changes in the transmitted power at a specific wavelength, thus a high responsivity. Moreover, since this component is made of pure silica, which softens at $T \sim 1680{ }^{\circ} \mathrm{C}$, it can potentially reach very high temperatures.

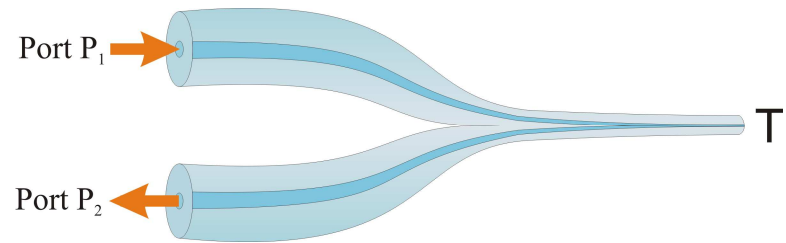

Fig. 1 Schematic of a micrometric fiber coupler tip (MFCT). Light is injected from port $\mathrm{P}_{1}$ and collected from port $\mathrm{P}_{2}$.

\section{Response time}

The device response time is related to its mass and thermal properties. If $\alpha, c$ and $\rho$ represent the surface heat transmission coefficient, heat capacity and density of the MFCT in the region where the surrounding fluid has temperature $T_{f}$, its dynamic thermal behavior is described by:

$$
\alpha A\left(T_{f}-T\right) d t=c \rho V d T
$$

where $V$ and $A$ are the volume and surface area of the heated region. The integration of this equation shows that the time constant (thus response time) of the device is given by:

$$
\tau=\frac{c \rho V}{\alpha A}=\frac{c \rho}{\alpha} \frac{r}{2}
$$

and it is inversely proportional to the MFCT radius $r$. Assuming $\alpha=10 \mathrm{~W} / \mathrm{m}^{2} \mathrm{~K}$ (natural convection), $c=0.8 \mathrm{~kJ} / \mathrm{kgK}$, $\rho=2300 \mathrm{~kg} / \mathrm{m}^{3}$ and $r=1.25 \mu \mathrm{m}$, the device response time results to be $\tau=0.12 \mathrm{~ms}$. Because of the small size, thus heat capacity, this device exhibits extremely fast response times. The response time of the packaged device will then be limited by the thermal properties of the packaging. 


\section{Temperature dependence of the MFCT}

The MFCT spectral response can be predicted by assuming that power exchange at the output ports occurs mainly as a result of the interference between the lowest-order symmetric and antisymmetric supermodes of the waveguide formed by the whole cross section in the minimum waist region. Because of the low manufacturing temperature, the MFCT can be assumed to be a "weakly fused" coupler, approximated by two touching cylindrical waveguides (Fig. 2(a) shows a schematic of coupler cross section in this approximation) [8]. If $f$ is the power fraction reflected at the tip end, unpolarized light entering the input port $\mathrm{P}_{1}$ will result in normalized power at the output port $\mathrm{P}_{2}$ :

$$
P_{2}=\frac{1}{2} f\left\{1-\cos \left[\left(\overline{C_{x}}+\overline{C_{y}}\right) L\right] \cos \left[\left(\overline{C_{x}}-\overline{C_{y}}\right) L\right]\right\}
$$

where $L$ is the coupling length of MFC which includes the waist uniform region and the transition region and $\overline{C_{x}}$ and $\overline{C_{y}}$ are the values of coupling coefficients $C_{x}$ and $C_{y}$ averaged over the whole length where coupling occurs. If $n_{0}$ and $n_{1}$ are the surrounding environment and tip refractive indices, then $f$ can be approximated by:

$$
f=\left(\frac{n_{1}-n_{0}}{n_{1}+n_{0}}\right)^{2}
$$

Equations (3) and (4) show that the output power depends on the wavelength $\lambda$ (through $C_{x}$ and $C_{y}$ ) and on refractive index $n_{1}$, coupling length $L$ and coupler radial size $2 a$, which are temperature dependent. Simulations were carried out to evaluate the temperature dependence of the MFCT output spectrum assuming the refractive index of silica at $\lambda \sim 1530 \mathrm{~nm}$ at $T=26{ }^{\circ} \mathrm{C}, 471{ }^{\circ} \mathrm{C}$ and $828{ }^{\circ} \mathrm{C}$ to be $1.44444,1.44961$ and 1.45352 [9], respectively. The diameter of each microfiber in the uniform region and the length of the uniform region $(2.5 \mu \mathrm{m}$ and $3 \mathrm{~mm}$, respectively) were assumed to expand with an average coefficient $5.5 \times 10^{-7}{ }^{\circ} \mathrm{C}^{-1}$. Fig. 2(b) shows the output power variation at three different temperatures. This spectral change can be used in two ways for temperature measurements: 1) evaluating the wavelength shift at a fixed output power and 2) evaluating the power change at a fixed wavelength. Here the first mechanism is used. When $T$ is increased from $26{ }^{\circ} \mathrm{C}$ to $828^{\circ} \mathrm{C}$, the resonance peak shifts $15.5 \mathrm{~nm}$. The sensor responsivity $R$ is defined as the wavelength shift associated to a temperature change. In Fig. 3(b), $R \sim 19 \mathrm{pm} /{ }^{\circ} \mathrm{C}$.

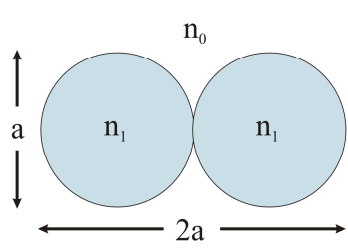

(a)

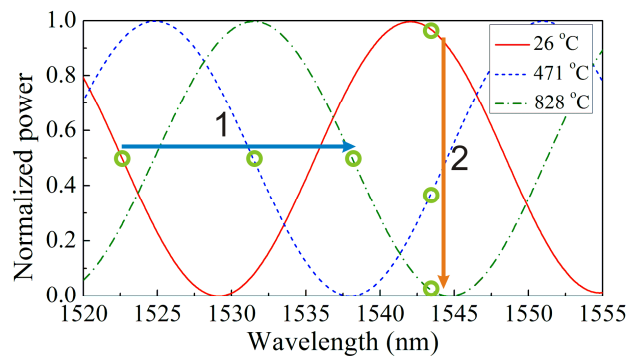

(b)

Fig. 3 (a) Schematic of the MCFT cross section in the "weakly fusing" approximation. (b) Normalized output power spectra at $T=26^{\circ} \mathrm{C}, 471^{\circ} \mathrm{C}$ and $828^{\circ} \mathrm{C}$. The blue and orange arrows show the two mechanisms which can be used for sensing: 1) wavelength change at constant normalized output power; and 2) normalized power change at fixed wavelength.

\section{Device fabrication and characterization}

The MFCT thermometer was manufactured by cutting into two equal parts a fiber coupler, fabricated using the microheater brushing technique [10] from two telecom optical fibers (SMF-28, Corning, USA) at a temperature of $T \sim 1450{ }^{\circ} \mathrm{C}$. The lengths of tapered and uniform waist regions were $\sim 25 \mathrm{~mm}$ and $\sim 6 \mathrm{~mm}$, respectively. A minimum waist radius of $r \sim 1.25 \mu \mathrm{m}$ was chosen to ensure high sensitivity, fast response and an adequate rigidity for the thermometer head, even if this resulted in slightly multimode guidance. In the MFCT, light launched from port $\mathrm{P}_{1}$ is partially reflected by the flat surface at the tip end and can be measured at port $\mathrm{P}_{2}$. Spectral shifts in the reflection dips are measured at the output port $\mathrm{P}_{2}$.

The sensor characterization was carried out using a microheater (NTT-AT, Tokyo, Japan) which can reach temperatures as high as $1500{ }^{\circ} \mathrm{C}$. The MFCT was inserted into the microheater center and reflection spectra were recorded at different $T$. The microheater temperature was changed by increasing the current flowing into the microheater from $0.4 \mathrm{~A}$ to $2.8 \mathrm{~A}$ in steps of $0.2 \mathrm{~A}$. Measurements were taken every 15 minutes to ensure a stable temperature. When the driver current is increased, the temperature increases and the peak redshifts to long 
wavelengths. An average $R \sim 11.96 \mathrm{pm} /{ }^{\circ} \mathrm{C}$ was achieved in the temperature range $\sim 247{ }^{\circ} \mathrm{C}$ to $\sim 1283{ }^{\circ} \mathrm{C}$, comparable to the value obtained for FBG thermometers at lower $T[11,12]$. The possible explanation for the difference between the theoretical calculation and experimental measurement could be the unperfected weakly fused coupler structure, the measured wavelength difference and the refractive index error. The sensor resolution was estimated to be $\sim 0.836$ ${ }^{\circ} \mathrm{C}$ for an OSA resolution of $0.01 \mathrm{~nm}$. The MFCT thermometer repeatability was measured recording spectra for increasing and decreasing temperatures at intervals of one hour. The wavelength shift with the temperature is shown in Fig. 4, showing that the MFCT thermometer has a good repeatability (within $3{ }^{\circ} \mathrm{C}$ ).

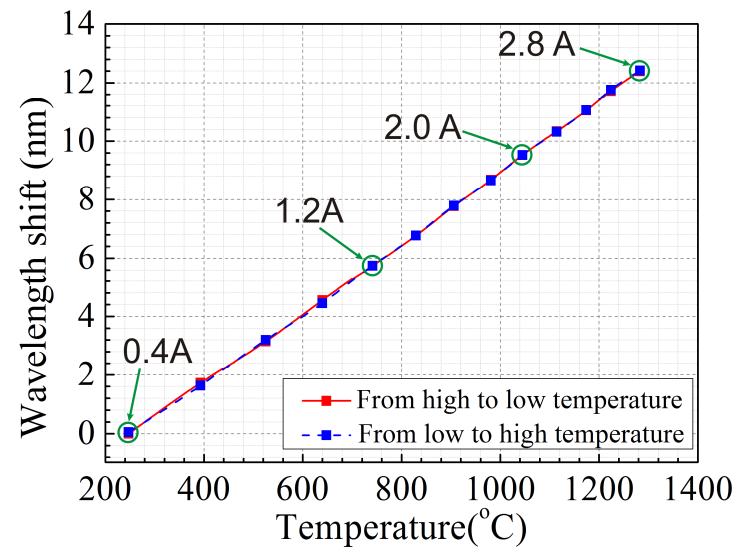

Fig. 4 Sensor characterization. The red solid curve and the blue dash curve report measurements for decreasing and increasing temperatures, respectively.

\section{Conclusions}

In summary, a fast compact sensor which uses a micrometer size optical fiber coupler tip for high- $T$ sensing has been demonstrated. This device exploits the temperature dependence of intermodal coupling in the coupler uniform waist region. This thermometer has a responsivity of $11.96 \mathrm{pm} /{ }^{\circ} \mathrm{C}$ and an operational range exceeding $1200{ }^{\circ} \mathrm{C}$ (from room temperature to $\sim 1283{ }^{\circ} \mathrm{C}$ ). Because of the small coupler cross section, response times are predicted to be the order of ms. Since the MFCT is made from pure silica and silica softens at $\sim 1680{ }^{\circ} \mathrm{C}$, this device could potentially work at temperatures well in excess of $1283^{\circ} \mathrm{C}$. MFCT offers several advantages, including compactness (few $\mu \mathrm{m}$ in diameter), high temperature measurement capabilities, high responsivity, fast response, easy connection with other fiberized optical components, simple fabrication and low cost.

\section{Reference}

[1] Y. Rao, "In-fibre Bragg grating sensors,” Meas. Sci. Technol. 8, 355-375 (1997).

[2] G. Coviello, V. Finazzi, J. Villatoro, and V. Pruneri, "Thermally stabilized PCF-based sensor for temperature measurements up to $1000{ }^{\circ} \mathrm{C}$," Opt. Express 17, 21551-21559 (2009).

[3] J. Kou, J. Feng, L. Ye, F. Xu, and Y. Lu, "Miniaturized fiber taper reflective interferometer for high temperature measurement," Opt. Express 18, 14245-14250(2010).

[4] D. Grobnic, S. J. Mihailov, C. W. Smelaser, and H. Ding., "Sapphire fiber Bragg grating sensor made using femtosecond laser radiation for ultrahigh temperature applications," Photonics Technology Letters, IEEE 16, 2505-2507 (2004).

[5] J. Feng, M. Ding, J.-L. Kou, F. Xu, and Y. Q. Lu, “An optical fiber tip micrograting thermometer,” IEEE Photonics J. 3, 810-814 (2011).

[6] J. Kou, S. Qiu, F. Xu, and Y. Lu, "Demonstration of a compact temperature sensor based on first-order Bragg grating in a tapered fiber probe,” Opt. Express 19, 18452-18457 (2011).

[7] H. Guo, F. Pang, X. Zeng, N. Chen, Z. Chen, and T. Wang, "Temperature sensor using an optical fiber coupler with a thin film,” Appl. Opt. 47, 3530-3534 (2008).

[8] F. P. Payne, C. D. Hussey, and M. S. Yataki, "Polarisation analysis of strongly fused and weakly fused tapered couplers", Electronics Lett. 21, $561-563(1985)$

[9] J. H. Wray and J. T. Neu, "Refractive index of several glasses as a function of wavelength and temperature," J. Opt. Soc. Am. 59, 774-776 (1969).

[10] G. Brambilla, E. Koizumi, X. Feng, and D. J. Richardson, “Compound-glass optical nanowires,” Electron. Lett. 41, 400-402 (2005).

[11] K. O. Hill and G. Meltz, "Fiber Bragg grating technology fundamentals and overview,” J. Lightwave Technol. 15, 1263-1276 (1997).

[12] A. D. Kersey, M. A. Davis, H. J. Patrick, M. LeBlanc, K. P. Koo, C. G. Askins, M. A. Putnam, and E. Joseph Friebele, "Fiber grating

sensors," J. Lightwave Technol. 15, 1442-1463 (1997). 